\title{
Investigation And Complexity Analysis of A Spatial Audio Codec Based on A Programmable Media-Processor
}

\author{
Chia-Hao Chang ${ }^{1}$, Jin-Hau Kuo ${ }^{2}$, Chi-Hao $\mathrm{Wu}^{1}{ }^{\mathrm{Ja}}$-Lin $\mathrm{Wu}^{1,2}$, Senior Member, IEEE \\ The Communications and Multimedia Laboratory, \\ ${ }^{1}$ Graduated Institute of Networking and Multimedia \\ ${ }^{2}$ Department of Computer Science and Information Engineering, \\ National Taiwan University, Taipei, Taiwan, ROC
}

\begin{abstract}
This paper presents a complexity analysis of a spatial audio coding scheme (known as MPEG surrounding) based on a commercially available programmable device. The analysis mainly focuses on the complexity of spatial audio cue analysis and synthesis, especially for the additional special tools used during the spatial cue synthesis stage. The analysis results can be a reference during building up a SAC codec on a DSP.
\end{abstract}

\section{INTRODUCTION}

With the fast development of newly-emerged computer technologies, digital audio video entertainment equipments are affordable and getting more and more popular. Numerous demands for $\mathrm{HD}$ (high definition) display device and the corresponding high quality video and audio digital contents are on the growth. All kinds of audio coding schemes are now in widespread use in multimedia applications. More recently, the trend of information technology (IT) converges toward the digital home, and undoubtedly, consumer electronic devices dedicated to entertainments will hold a significant position in everybody's home. Meanwhile, along with the prevalence of high quality but cheaper multi-channel loudspeaker devices, next generation digital multimedia content with high quality but low bit-rate multi-channel audio is surely to be the main stream in the near future. Now, building up a good multichannel sound reproduction environment at home has become more practicable and fashionable. This trend is driven by generic movie sounds and more and more multi-channel DVD movies. Furthermore, lots of new multi-channel applications emerged in these years. For example, mobile applications, portable devices, and automotive applications, such as car audio playback setups, tend to have the demand of multichannel audio playback. A carefully designed audio coding mechanism for gaining best surround performance, is therefore, of interest.

\section{THE MPEG STANDARD}

The demands for multi-channel audio contents can be served by burgeoning media content types, such as SA-CD and DVD-Audio. On the other hand, for bandwidth-

1. The Philips TriMedia processor IC is commercially available as the TM1500. constrained and multi-channel applications, the MPEG-2/4 Advanced Audio Coding (AAC) scheme has been developed as an efficient multi-channel audio format. The AAC provides excellent sound quality at bit-rates in the range of 256$320 \mathrm{kbit} / \mathrm{s}$ for 5-channel materials. Furthermore, MPEG organization now is building up a work plan about spatial audio coding (SAC) [1]. The work plan mainly focuses on:

\section{1) An extremely compact representation of the multi-channel} signal.

2) An inherent ability to present either the multi-channel signal to new equipment or the e.g. 2-channel signal to legacy equipment.

The MPEG committee has issued a call for proposal (CfP) for spatial audio coding (SAC) [2]. This will be an extension to audio codec for multi-channels. FhG/Agere [3], Dolby [4], Coding Technologies/Philips [5], and Panasonic [6] have submitted their proposals in response to the call. These proposals are based on parametric stereo [7] and BCC (binaural cue coding) [8]. MPEG now has defined the reference model 0 (RM0) for the standardization process. It is a combination of proposals from $\mathrm{FhG} /$ Agere and $\mathrm{CT} / \mathrm{Philips}$ [9]. The infrastructure of the generic spatial audio coding technology is shown in Fig. 1, 2, 3 .

\section{THE COMPLEXITY ANALYSIS}

As mentioned above, the main technique of $\mathrm{SAC}$ is $\mathrm{BCC}$ (Binaural cue coding). $\mathrm{BCC}$ enables parametric representations of a spatial audio and delivers multi-channel output (with an arbitrary number of channels) from a single audio channel plus some side information. It uses parameters like inter-channel time difference, inter-channel level difference and inter-channel coherences, and so forth to support surround sound (such as 5.1 channels) at very low bitrates. Lately the "MP3Surround" [11] technology from FhG uses this technique to add surround-sound capabilities to the MP3 algorithm at the cost of few additional bits. This technique can also be used as parametric stereo to support just two channels for backward compatibility.

Recently, the MPEG committee releases their spatial audio coding RM0 reference software. According to the reference software and its architecture detail [12], we mainly focus on the complexity analysis of the MPEG RM0 reference software 
on a programmable media processor ${ }^{1}$. We analyzed the complexities of the following stages:

1) Audio source down-mix stage,

2) Audio cue estimation stage,

3) Audio cue synthesis stage

Based on the complexity analysis results, we can further implement the low cost media processor based spatial audio encoder/decoder. Fig. 4 and fig. 5 show the functional profiles of the encoding and decoding process, respectively.

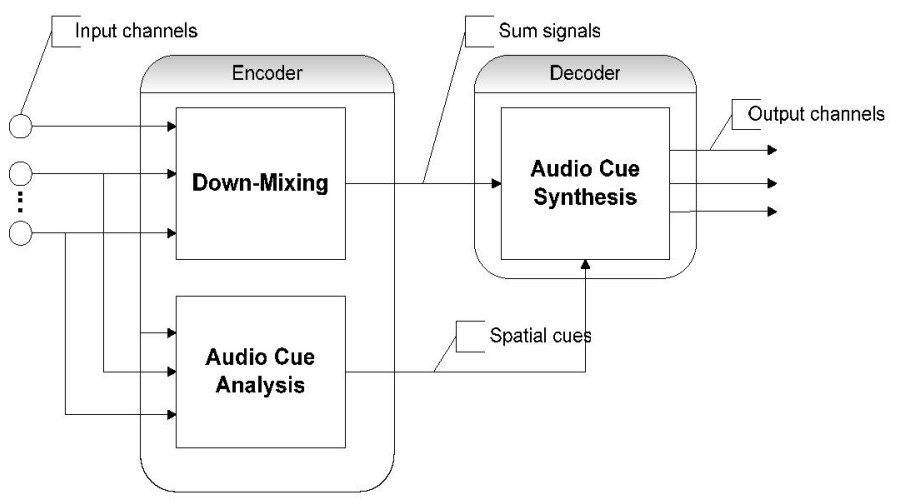

Fig. 1. Generic spatial audio coding scheme.

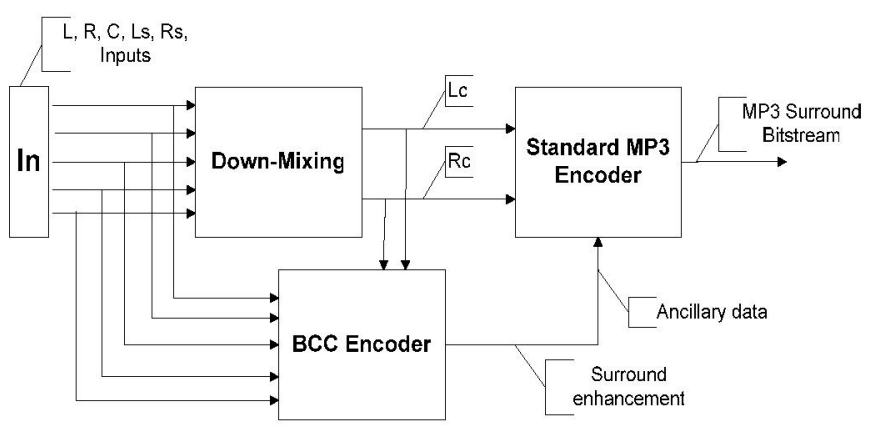

Fig. 2. The structure of a spatial audio coding encoder.

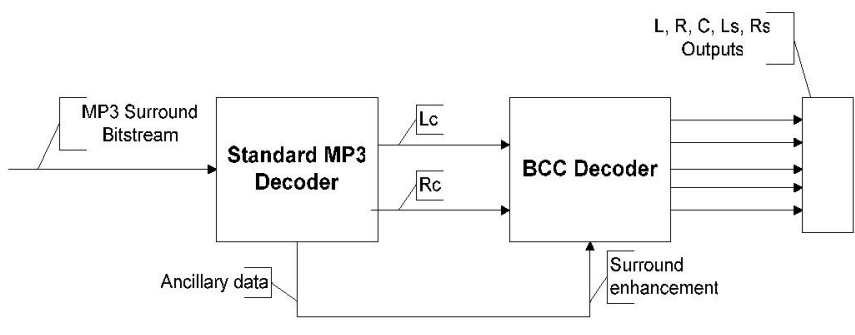

Fig. 3. The structure of a spatial audio coding decoder.

\begin{tabular}{|c|c|c|}
\hline Functions & Total Cycles & Percentage \\
\hline $\begin{array}{l}\text { Hybrid QMF filtering } \\
\text { Including|: }\end{array}$ & 168666258 clocks & $92.280 \%$ \\
\hline Interleaue & 258069 clock5 & $0.141 \%$ \\
\hline QNF filtering & 67852891 clocks & $37.123 \%$ \\
\hline Hybrid filtering & 161555.298 clock5 & $55.015 \%$ \\
\hline $\begin{array}{l}\text { Calculate cues and dounmix } \\
\text { Inverse hybrid filtering }\end{array}$ & $\begin{array}{r}2457478 \text { clocks } \\
11652591 \text { clocks }\end{array}$ & $\begin{array}{l}1.345 \% \\
6.375 \%\end{array}$ \\
\hline Total functions & 182776327 clocks & $100.00 \%$ \\
\hline
\end{tabular}

Fig. 4. The profile of a spatial audio coding encoder.

\begin{tabular}{|c|c|c|}
\hline Functions & Total Cycles & Percentage \\
\hline Decode spatial cues & 74052 clocks & $0.020 \%$ \\
\hline MDGT to QMF & 1921 clocks & $5.001 \%$ \\
\hline Hybrid QMF analysis & 1630912 a clocks & $4.392 \%$ \\
\hline Parameter smoothing & 34425 clocks & $0.099 \%$ \\
\hline Greate $x$ matrix & 237982 clocks & $5.064 \%$ \\
\hline Hake M1 and H2 matrix & 796153 clocks & $0.214 \%$ \\
\hline Apply H1 matrix & 68849260 clocks & $18.540 \%$ \\
\hline create $W$ matrix & 26650271 clocks & $7.177 \div$ \\
\hline Apply H2 matrix & 8891945.2 clocks & $23.045 \%$ \\
\hline TP Process & 169475269 clocks & $45.638 \%$ \\
\hline Total Functions & 3713470105 clocks & $160.00 \%$ \\
\hline
\end{tabular}

Fig. 5. The profile of a spatial audio coding decoder.

\section{REFERENCE}

[1] J. Herre, C. Faller, S. Disch, C. Ertel, J. Hilpert, A.Hoelzer, K. Linzmeier, C. Spenger, P. Kroon "Spatial Audio Coding: Next-Generation Efficient and Compatible Coding of Multi-Channel Audio", 117th AES Convention, San Francisco 2004, Preprint 6186.

[2] ISO/IEC JTC1/SC29/WG11 MPEG, Document N6455, "Call for Proposals on Spatial Audio Coding", Munich 2004.

[3] Jürgen Herre, Christof Faller, C. Spenger, J. Hilpert, K. Linzmeier. ISO/IEC JTC1/SC29/WG11 MPEG2004, M11075. "Fraunhofer/Agere Submission to Spatial Audio CfP", July 2004

[4] Mark Vinton, Mark Davis, Matt Fellers, Grant Davidson. ISO/IEC JTC1/SC29/WG11 MPEG2004, M11090. "Dolby Laboratories Submission to CfP on MPEG-4 Spatial Audio Coding", July 2004

[5] ISO/IEC JTC1/SC29/WG11 MPEG2004, M11001. "CT/Philips contribution to CfP on spatial audio coding", July 2004

[6] Naoya Tanaka, Kok-Seng Chong, Ching-Hua Ng, Yoshiaki Takagi, Kojiro Ono, Mineo Tsushima,. ISO/IEC JTC1/SC29/WG11 MPEG2004, M1 1001. "Technical Description and Performance Test Results of Panasonic Spatial Audio Coding", July 2004

[7] E. Schuijers, J. Breebaart, H. Purnhagen, J. Engdegård "Low complexity parametric stereo coding", Proc. 116th AES convention, Berlin, Germany, 2004, Preprint 6073.

[8] C. Faller and F. Baumgarte, "Binaural Cue Coding-Part II: Schemes and applications," IEEE Trans. on Speech and Audio Proc., vol. 11, no. 6, Nov. 2003.

[9] ISO/IEC JTC1/SC29/WG11 MPEG, Document N6814, "Workplan for MPEG-4 Spatial Audio Coding", Palma de Mallorca 2004.

[10] http://www.iis.fraunhofer.de/amm/download/mp3surround/

[11] J. Herre, H. Purnhagen, J. Breebaart, C. Faller, S. Disch, K. Kjörling, E. Schuijers, J. Hilpert, F. Myburg "The Reference Model Architecture for MPEG Spatial Audio Coding", Proc. 118th AES convention, Barcelona, Spain, 2005, Preprint 6447. 